Lousiana State University), and the fate of spilled oil (R. A. Larson, Stroud Water Research Centre, Avondale, Pa.). More than 300 people from a small community attended and there was active questioning and discussion. This might be a possible way for scientists to bring their scientific work and opinions directly, rather than through the media, to a lay audience, especially on controversial topics.

\section{Thin film technology}

from C. W. Pitt

The first of an intended series of international conferences on ionassisted thin film coating techniques was held in Edinburgh on 8-10 June 1977. The Proceedings are available from CEP Consultants, Edinburgh. The next IPAT conference will be held in London in July 1979.

THE presentations covered a wide range of experimental and production processes, ranging from ion plating, ionised clusterbeam technology, r.f. sputtering, magnetron sputtering, sputter-ion plating, ion implantation and activated reactive evaporation and plasma deposition. An equally large range of applications of the various methods was presented including tribology, tool coatings, corrosion resistant turbine and airframe components, decorative finishes, gas storage, semiconductor, optical and solar devices, electronic components and steel hardening.

A number of the papers were of particular interest to electrical and electronic engineers and solid-state physicists. The paper by T. Takagi et al. (Kyoto University) on ionised cluster-beam technology suggested that the required incoming material mobility on the substrate can be achieved by partially ionising clusters of atoms from an adiabatic expansion source and, provided that the deposition rate is maintained at least $10^{3}$ higher than the residual gas impingement rate, single crystal and near bulk density films of a wide range of materials may be deposited. The technique has been applied in numerous ways-for example, epitaxial silicon on silicon, InSb on sapphire, GaAs on $\mathrm{Cr}$ : GaAs. Silicon solar cells grown $\mathrm{n}$ on $\mathrm{p}$, have been fabricated with exposed-surfacelayers of $140 \AA$ and which exhibit $70 \%$ of the peak output voltage from $450 \mathrm{~nm}$ to $950 \mathrm{~nm}$. Low resistance noble metal films deposited on glass substrates have

C. W. Pitt is Lecturer in Electronics at University College, London. demonstrated near bulk resistivity in films $200 \AA$ thick; thicker films of the same metals have been used as interconnections on passivated semiconductor chips and have successfully covered $8 \mu \mathrm{m}$ oxide steps. Furthermore, ohmic contacts have been made to semiconducting material (Ag on n-type silicon, $\mathrm{AuBe}$ on p-type GaP) without interface metals or sintering. Ionised clusterbeam deposition on $\mathrm{Mn}$-doped $\mathrm{ZnS}$ was also reported to have produced d.c. electroluminescent displays of similar properties to those produced by evaporation and subsequent implantation of the dopant. The superior adhesion properties of the process were demonstrated by the fabrication of flexible printed circuit board which meets the requirements of IPC-FC-240B specification.

K. Jones et al. (ICI, Runcorn) have also examined $\mathrm{Au}, \mathrm{Cu}$, and $\mathrm{Al}$ layers deposited on several types of flexible plastic substrates-polyethersulphone, polypropylene, polyimide and polyester. They concluded that the $\mathrm{Cu}$ : polyethersulphone system was particularly suited for flexible pcb construction by the ion-plating process. lon plating produced good decorative finishes for most of the metal/plastic combination. Sims, in a review of ionplating applications, again mentioned the fabrication of printed circuit board material. He also cited a number of less obvious applications such as battery-grid coatings which result in an improvement in the energy storage efficiency of lead/acid cells, aluminium coatings for steel and plastic lampreflectors and which are subsequently protected by ion-plated glassy layers, and also coatings for X-ray anodes for use in the soft wavelength spectrum. B. Heinz (Leybold Heraeus, Hanau) reported the use of electron-beam ion plating for forming the contact layers of noble metals on reed-relay blades. $\mathrm{He}$ and his colleagues have found that the switching lifetime of the plated blades could be enhanced by a factor of 10 times in appropriate conditions, and that the process produced blades with reduced sticking probability and contact resistance. The process is in industrial production.

Anti-reflective and protective coatings for germanium lenses for infrared imaging were discussed by E. Henderson (Pilkington, St Asaph). Although the experiments reported were in the early stages, it was clear that serious consideration is being given to the use of ion plating for depositing $\mathrm{ZnS}$ antireflection coatings on five-element large-area zoom lenses for operation at $8-12 \mu \mathrm{m}$ in corrosive atmospheric conditions, in aircraft (for example). Preliminary data indicate an improvement in lifetime of four to eight times in adverse conditions. C. W. Pitt (University College, London) reviewed the r.f. sputtering process, with particular emphasis on the capability of this process for depositing dielectric films with good optical properties. The technique has been found to be attractive for fabricating optical waveguiding components for integrated-optics applications. Hard graphitic carbon deposits may be prepared by vapour deposition from a hydrocarbon gas excited into a plasma-phase by an r.f. field S. M. Ojha (University of Sussex) presented some details of this process and of the films produced-it was projected that the rather impressive hardness (unscratched by tungsten carbide) and chemical passivity (insoluble in any of the solvents and acids used, including hydrofluoric acid) might lead to applications in semiconductor passivation and infrared filters.

The increasing use of nuclear power stations for generating electricity has produced several contentious issues, not least of which is the disposal of radioactive wastes. The storage of active gases is particularly sensitive in view of the rapid dispersal if the container is damaged. D. S. Whitmell et al. (UK Atomic Energy Authority) have devised an ingenious method of containing radioactive ${ }^{85} \mathrm{Krypton}$ for the required $100-200 \mathrm{yr}$ and which greatly reduces the potential danger if the container is damaged. The gas is incorporated into a copper thin film matrix by plating a thin film of the metal on a substrate by d.c. sputtering, followed by a reversal of the field so that gas ions are bombarded at the deposited film, penetrate and are trapped. The process is then repeated many times until a thick deposited layer with occluded gas entrapment is built up. It was claimed that up to $5 \%$ (atomic) gas occlusion could be achieved-a similar storage capability to a high pressure gas cylinder (approximately 1701 of gas at NTP per 1 of metal). Damage to the deposit results in very little gas release and only from the area local to the damage.

Two papers on ion implantation, by C. Dearnley et al. (Atomic Energy Research Establishment, Harwell) and by V. Ashworth et al. (University of Manchester Institute of Science and Technology) suggest that semiconductor devices are not the only area in which the electronic/electrical industry will be influenced by this process. The papers primarily dealt with ion implantation of dopants into metals as a means of altering the wear-resistance of the host metal. But it was apparent from the results of tests on the electrochemical potentials of the doped materials that cathodic protection with improved efficiency may well be feasible. 